\title{
The Role of Compassion for Self and Others, Compassion Fatigue and Subjective Happiness on Levels of Well-Being of Mental Health Professionals
}

\author{
Archontia Mantelou, Eirini Karakasidou \\ Institution for Counseling and Psychological Studies (ICPS College), University of Central Lancashire, Athens, Greece \\ Email: andymantelou@hotmail.com
}

How to cite this paper: Mantelou, A., \& Karakasidou, E. (2019). The Role of Compassion for Self and Others, Compassion Fatigue and Subjective Happiness on Levels of Well-Being of Mental Health Professionals. Psychology, 10, 285-304.

https://doi.org/10.4236/psych.2019.103021

Received: December 4, 2018

Accepted: February 18, 2019

Published: February 21, 2019

Copyright $\odot 2019$ by author(s) and Scientific Research Publishing Inc. This work is licensed under the Creative Commons Attribution International License (CC BY 4.0).

http://creativecommons.org/licenses/by/4.0/

\section{(c) (i) Open Access}

\begin{abstract}
This study aimed to explore the role of compassion for self and others, compassion fatigue and subjective happiness on levels of well-being of mental health professionals as well as to contribute new knowledge by investigating whether and to what extent subjective happiness of therapists can be predicted. A sample population of the online survey comprised 58 mental health professionals living and working in Greece-Athens and the method of recruitment was by opportunity sampling. This particular study employed a series of two-tailed Pearson $r$ correlation analyses as well as independent samples t-test and a hierarchical multiple regression analysis for the analysis of the data. The results that revealed through the four different questionnaires (Self-Compassion Scale, Professional Quality of Life Scale, Compassion-for-Others-Scale and Subjective Happiness Scale) showed that self-compassion has a protective function for the therapists. It enhances their subjective happiness, which in turn protects mental health and professional quality of life of therapists by reducing the negative effects of compassion fatigue. These findings highlight the importance of self-compassion, compassion for others and subjective happiness among therapists, as they are correlated with compassion fatigue.
\end{abstract}

\section{Keywords}

Compassion for Self and Others, Compassion Fatigue and Subjective Happiness, Mental Health Professionals

\section{Compassion}

Self-compassion, as defined by Neff (2003a), concerns being kind, warm and 
standing with understanding toward yourself when you suffer, fail or feel inadequate, rather than criticizing and blaming yourself or ignoring the pain and negative feelings. This pain can come either from the external conditions of life, when they are painful, or from the actions of the person, the failures or personal weaknesses (Neff, 2009a). The concept of self-compassion entails three basic interacting components: self-kindness and understanding towards negative aspects of the self versus self-judgment, common humanity versus isolation, which means the view of the defect or the negative experiences experienced by the individual as part of the overall human experience as well as mindfulness versus over-identification which means the preservation of painful thoughts and emotions in a conscious awareness, an emotional balance and peace even when the person is facing difficult situations (Barnard \& Curry, 2011; Neff, 2003a).

Self-compassion is not only a positive concept but has been positively correlated with many concepts of positive psychology, such as positive feelings and positive characteristics of the individual (Neff, Kirkpatrick, \& Rude, 2007). In particular, high levels of self-compassion are associated with high levels of happiness, life satisfaction, optimism, curiosity, wisdom, social connection, personal motivation for necessary changes in one's life, emotional intelligence, kindness, peace, while through self-compassion the individual is helped to find hope and meaning in the difficulties of life (Heffernan, Griffin, McNulty, \& Fitzpatrick, 2010; Neff, 2003a; Neff, 2003b; Neff, 2009a; Neff, Kirkpatrick, \& Rude, 2007). Overall, the approach of painful emotions in a self-compassionate way seems to facilitate the development, exploration and a wiser way of seeing oneself and others (Neff, Kirkpatrick, \& Rude, 2007).

Compassion for self is an important factor in understanding mental health as it appears to play an important role in the way people handle unpleasant and negative events (MacBeth \& Gumley, 2012; Neff, 2003a). More specifically, it has been found that people with high levels of self-compassion react with less intensity to negative events, make more balanced and less negative reviews of their experiences, and experience no negative feelings for themselves when they think of stressful or negative situations (Leary, Tate, Adams, Allen, \& Hancock, 2007; Neff, Kirkpatrick, \& Rude, 2007). Additionally, it has been found that individuals with high levels of self-compassion deal more effectively stressful life events such as divorce (Sbarra, Smith, \& Mehl, 2012) and chronic pain (Wren, Somers, Wright, Goetz, Leary, \& Keefe, 2012). The above research findings support the hypothesis that self-compassion can facilitate more healthy management of stressful states (Leary et al., 2007; Neff, 2003a).

\subsection{The Concept of Compassion Fatigue to Therapists' Mental Health}

The practice of counseling and psychotherapy can be described as a psychologically demanding profession. The fact that the therapeutic work with patients with psychological difficulties requires care, combined with the fact that therapists usually do not pay much attention to self-care, as they are trained and spend 
a lot of time on how to care for others, seems psychologically affect the professionals (Dunkley \& Whelan, 2006; Figley, 2002; Skovholt, Grier, \& Hanson, 2001; Smith \& Moss, 2009). In particular, aggravating factors pertaining to therapists themselves include the trauma history of the therapist, the absence of a supportive network, the lack of experience, the difficulty in maintaining emotional boundaries and the hyper-engagement with their servants (Adams, Matto, \& Harrington, 2001; Dunkley \& Whelan, 2006; Rupert \& Morgan, 2005; Skovholt \& Ronnestad, 2003).

The above stressors can lead to harmful effects on the mental health of professionals and their ability to work effectively (Barnett, Baker, Elman, \& Schoener, 2007). The concept of compassion fatigue, generally refers to a decrease in the capacity or interest in the therapist's suffering the pain of others and is a result of the stress resulting from the exposure of the professional to extremely stressful or traumatic events through his work with people who have experienced (Adams, Boscarino, \& Figley, 2006; Bride \& Figley, 2007; Figley, 2002). Fatigue of compassion includes two parts that of burnout and secondary traumatic stress, as it may appears with symptoms of burnout and/or with symptoms of secondary traumatic stress (Stamm, 2010).

More specifically, burnout syndrome involves emotional exhaustion, depersonalization, and reduced personal sense of accomplishment and leads to mental and professional harm, as professionals are unable to effectively practice their clinical work (Rosenberg \& Pace, 2006). Regarding secondary traumatic stress, this refers to the professional's reaction to the traumatic and stressful material of his or her patients and includes symptoms such as feelings of fear, sleep disturbances, annoying thoughts and images, and avoid situations recalling the traumatic event (Stamm, 2005).

\subsection{The Concept of Compassion for Self and Others to Therapists}

Although mental health professionals express interest and compassion in their services, this does not necessarily mean that this compassionate attitude is directed towards themselves (Germer \& Neff, 2013). Self-compassion is a relatively new concept and therefore has been little studied with therapists and mental health professionals in general (Grant \& Kinman, 2012; MacBeth \& Gumley, 2012). Patsiopoulos and Buchanan (2011) have studied qualitatively the concept of self-compassion in counselors and explored the ways with which they show self-compassion, with the results showing that counselors act self-compassionate by adopting an attitude of kindness towards oneself, mindfulness of present experience and authenticity.

The few relevant researches that have been conducted have shown the importance of focusing on both the mental health of therapists as well as in counseling and psychotherapy, as it is argued that self-compassion improves the mental health of the therapists, their work efficiency and their therapeutic relationship with their servants (Patsiopoulos \& Buchanan, 2011). In particular, it has been found that self-compassion displayed by counselors is negatively correlated with 
work-related stress, with compassion fatigue and with professional burnout, while positively correlated with compassion satisfaction (Beaumont, Durkin, Hollins Martin, \& Carson, 2016; Ringenbach, 2009). In addition, it has been shown that self-compassion can increase the chances of therapists engaging in self-care activities, facilitating the adoption of an attitude with care and self-esteem (Neff, 2003a; Patsiopoulos \& Buchanan, 2011).

Self-compassion also seems to play an important role in the treatment itself, since therapists who act in a self-compassionate manner, encourage their servants to behave in a similar self-compassionate way and thus the therapeutic work becomes more authentic, human-centered and less based on techniques (Boellinghaus, Jones, \& Hutton, 2013). Also, Patsiopoulos and Buchanan (2011) found, in their qualitative study, that self-compassion has a positive effect on the ability of therapists to work effectively, helping them to have less unrealistic expectations, develop more effective boundaries and find the balance between the needs of the clients and their own and consequently correct themselves, when necessary.

The above studies, which investigated self-compassion in therapists, have shown that it is associated with less negative and more positive effects on their mental health (Patsiopoulos \& Buchanan, 2011; Ringenbach, 2009). In this respect, self-compassion can be considered as a factor that can promote subjective happiness of the therapists, since the existing literature studying the core effects of self-compassion on general population, indicate the high levels of subjective happiness for those with compassionate attitude (Heffernan, Griffin, McNulty, \& Fitzpatrick, 2010; Neff, 2003a; Neff, 2003b; Neff, 2009a).

\subsection{The Concept of Subjective Happiness}

Subjective happiness, is characterized as the psychological state of wellbeing, joy, and contentment (Lyubomirsky, 2001). The definition of subjective happiness is consisted of three major components: the positive affect in experiencing positive emotions such as interest, joy and excitement, the negative affect that reflects in experiencing emotions that cause discomfort such as anger, hate, fear, guilt and sadness as well as the life satisfaction which involves cognitive evaluations of an individual about various areas of his life (Kumcagiz \& Gunduz, 2016).

More specifically, according to Krieger and colleagues' (2015) research, there is a positive correlation between self-compassion and positive affect and a negative correlation between self-compassion and negative affect (Neff, Rude, \& Kirkpatrick, 2007; Neff \& Vonk, 2009). In other words, individuals with higher levels of self-compassion seem to have higher positive affect and lower negative affect and can thus cope more effectively with conditions that are stressful in everyday life, in comparison to those lacking self-compassion. So, a person who exhibits high levels of subjective happiness will come up with a positive affect. This is known as positive affectivity and is referred to a trait that describes the different intensity of positive emotions. Moreover, a vast number of studies has reached to the conclusion that self-compassion is a factor that is in correlation 
with positive mind-states, as for example life satisfaction and is a force working towards its improvement (Neely et al., 2009; Seligowski, Miron, \& Orcutt, 2014; Van Dam, Sheppard, Forsyth, \& Earleywine, 2011; Wei et al., 2011; Yang, 2016). Although there has been an increase of interest from researchers in what makes people happy (Myers \& Diener, 1995) research indicates that the term subjective happiness is used to describe the idea of getting the important things that someone desires in life, as well as specific pleasurable effects that normally go along with this belief (Kraut, 1979).

\section{The Importance of This Particular Research}

In the existing literature there is a plethora of research into the harmful effects that can arise as a result of counseling and psychotherapy and influence the mental wellbeing of the therapists and hence the quality of their work (Barnett, Baker, Elman, \& Schoener, 2007). Nevertheless, there are few investigations for the concept of self-compassion in mental health professionals. Regarding the relationship between self-compassion and subjective happiness, there is a lack of researches that explore their relationship to both the general population and mental health professionals. Concerning the relationship between subjective happiness and the variables of compassion for self and others and compassion fatigue, no prediction relationships, between them, have been examined. This study attempts to fill the existing gap in the literature by studying the relationship of the above variables to a sample of Greek therapists.

Beyond the gap in the literature, the importance of investigating the relationships between the above variables lies in the fact that mental health professionals undertake the demanding and potentially stressful task of caring for people experiencing psychological difficulties, which adversely affects not only their psychological well-being but also their ability to work effectively, as it has been shown that the mental health of the therapists ensures the quality of their work and their therapeutic interventions (Barnett et al., 2007). More specifically, it has been found that the effectiveness of therapeutic interventions by professionals may be negatively affected by their attempt to maintain their personal and professional wellbeing and therefore may find it difficult to maintain the appropriate limits, to show empathy and to work within the framework ethics (Dunkley \& Whelan, 2006; Lawson \& Myers, 2011). In terms of fatigue of compassion, has been shown to lead to personal and professional harm to therapists (Figley, 2002). Consequently, studying those factors that may be associated with reduced levels of compassion fatigue and can positively contribute to promoting the psychological well-being of professionals by protecting them from the negative effects their work can bring is particularly important for both therapists themselves and their patients.

\section{Research Question and Hypotheses}

The purpose of this study is linked with the relationship between self-compassion, 
compassion for others, compassion fatigue, and subjective happiness for mental health specialists, who work as therapists. This is based on the overall aim of promoting the psychological well-being of mental health professionals and ensuring the quality of their work by highlighting the factors and processes involved. There is also the need for therapists' self-care practices which will be revealed through the exploration of the relationship between self-compassion, compassion for others, compassion fatigue, and subjective happiness. More specifically, this study aims to study the possible correlations between subjective happiness, self-compassion, compassion for others, and fatigue of compassion among mental health specialists. In addition, the present study explores whether and to what extent subjective happiness of therapists can be predicted. It also examines whether there are differences between several demographic variables (age, gender, therapeutic approach, work experience etc.) and compassion fatigue, self-compassion and compassion for others.

Based on the existing theory and previous research, the assumptions of this research are as follows:

1) It is hypothesized a positive correlation between self-compassion and mental health professionals' subjective happiness.

2) It is hypothesized a negative correlation between compassion fatigue and variables of self-compassion, compassion for others and subjective happiness of therapists.

3) Which variables can predict subjective happiness?

4) The differences between several demographic variables (age, work experience, number clients, personal therapy) and compassion fatigue, self-compassion and compassion for others.

\section{Method}

\subsection{Design}

This particular study employed a series of two-tailed Pearson $r$ correlation analyses as well as independent samples t-test and a hierarchical multiple regression analysis for the analysis of the data. The tested variables of the study included self-compassion, compassion fatigue, compassion for others and subjective happiness of the therapists.

\subsection{Participants}

In the current research, a sample population comprised 58 mental health professionals living and working in Greece-Athens and the method of recruitment was by opportunity sampling. Therapists originated from a variety of fields in mental health such as psychology, psychiatry, social work, counseling, and were either practitioners or trainee therapists. The prerequisite for their participation in the research was the implementation of counseling or psychotherapy, either in a public or private context. More specifically, the sample of the study consisted of 54 women (93.1\%) and 4 men (6.9\%) and they ranged in age from aged 20 to 64 
years old with mean age $38(\mathrm{SD}=10.86)$. The sample was also consisted of 26 psychologists (44.8\%), 26 mental health counselors (44.8\%), and 6 psychiatrists and social workers (10.3\%). Regarding the level of education of the participants, $6(10.3 \%)$ had a basic degree, $50(86.2 \%)$ had postgraduate studies and $2(3.4 \%)$ were $\mathrm{PhD}$ holders. Regarding the theoretical approach of the participants, 2 (3.4\%) followed the systemic approach, $4(6.9 \%)$ the synthetic/eclectic, 4 (6.9\%) the psychoanalytic/psychodynamic, $6(10.3 \%)$ the cognitive-behavioral, $34(58.6 \%)$ the person-centered and 4 (6.9\%) the existential psychotherapy. The duration of the participants' professional experience ranged from 0 to 18 years $(\mathrm{M}=4.39, \mathrm{SD}=4.59)$ and the average of patients treated in a typical week was 8 . Finally, 28 participants had experienced personal psychotherapy in the past, while 28 of them attend psychotherapy in the present. Participation in the survey was voluntary, based on informed consent while the study was also conducted according to the British Psychological Society ethical guidelines for research.

\subsection{Materials}

Initially, participants were asked to respond to a demographic questionnaire (see for the demographic paper) that included information about their age, gender, education level, occupational status, theoretical approach, and the clinical experience of the respondent. In addition, there were questions about the average number of patients treated in a typical week as well as whether the participant attended personal psychotherapy. All participants also filled out a number of self-report measures which were distributed to the Greek versions.

Self-compassion Scale (SCS) (Karakasidou, Pezirkianidis, Galanakis, \& Stalikas, 2017; Neff, 2003a). Participants were given the 26-item Self-Compassion Scale (SCS, Neff, 2003a) that was provided in the Greek version translated and weighted in Greek population (Karakasidou, Pezirkianidis, Galanakis, \& Stalikas, 2017). The scale consists of 6 factors, three of which are indicative of the positive aspects of the concept and the other three refer to the negative qualities an individual maintains when he is not self-compassionate. These factors are self-kindness opposed to self-judgment, common humanity opposed to isolation and mindfulness opposed to over-identification (Neff, 2003b). The subscale of self-kindness (e.g., "I try to be understanding and patient toward aspects of my personality I don't like") includes 5 statements and presents acceptable internal consistency ( $\alpha=.78$ ). The subscale of self-judgment (e.g., "I'm disapproving and judgmental about my own flaws and inadequacies") includes 5 statements and presents an acceptable credibility of internal consistency $(\alpha=.77)$. The subscale of Common Humanity (e.g., "I try to see my failings as part of the human condition") includes 4 statements, which show good credibility of internal consistency $(\alpha=.80)$. The subscale of isolation (e.g." "When I think about my inadequacies it tends to make me feel more separate and cut off from the rest of the world") includes 4 statements demonstrating acceptable internal consistency $(\alpha=.79)$. The 
subscale of Mindfulness (e.g., "When something painful happens I try to take a balanced view of the situation") includes 4 statements with acceptable internal consistency ( $\alpha=.75$ ). The subscale of Over-Identification (e.g., "When I'm feeling down I tend to obsess and fixate on everything that's wrong") includes 4 statements and has good internal reliability $(\alpha=.81)$. The credibility of internal consistency for the 26 statements of the scale of self-compassion is excellent ( $\alpha$ $=$.92) (Neff, 2003b). Responses are given on a 5-point scale from "Almost Never" to "Almost Always". Mean scores on the six subscales are then averaged (after reverse-coding negative items) to create an overall self-compassion score ranging from 26 to 130 . Higher scores correspond to higher levels of self-compassion.

Professional Quality of Life Scale (ProQOL). The participants were given, particularly, the fifth revised version of the scale, which presents better psychometric properties than older versions (Stamm, 2010). This scale is the most widely used scale for measuring the professional quality of life of professionals working with people who have experienced stressful or traumatic events. The theory on which the tool is based is that the professional quality of life of the specialists, who provide support, includes a positive and a negative side. The positive aspect concerns the satisfaction from compassion and the negative aspect concerns the fatigue of compassion. The fatigue of compassion includes two parts, that of burnout and that of secondary traumatic stress (Stamm, 2010). The tool, therefore, consists of three scales, professional burnout, secondary traumatic stress and satisfaction of compassion, each of which includes 10 self-report statements rated on a 5-point Likert scale (1-never, 2-rare, 3-sometimes, 4-often, 5 -very often) and the respondent is asked to evaluate the extent to which each sentence matches its personal experience in the last month (Stamm, 2010).

The scale of compassion satisfaction reflects positive emotions and thoughts that the expert may experience in response to his work with the patients. Highest scores on this scale represent greater satisfaction of compassion. The scale has good credibility of internal consistency ( $\alpha=.88$ ) (Stamm, 2010). The scale of burnout refers to the part of the fatigue of compassion that is characterized by feelings of misery, lack of attachment and lack of sensitivity to work, and may involve exhaustion, pressure, and a sense of self-removal. Highest score on this scale means that the person is at higher risk for professional burnout. The scale presents acceptable credibility of internal consistency ( $\alpha=.75)$ (Stamm, 2010). The scale of secondary traumatic stress is the second component of fatigue of compassion and refers to the individual's reaction to the traumatic and stressful material. The scale shows good credibility of internal consistency $(\alpha=.81)$ (Stamm, 2010).

Compassion-for-Others-Scale (Pommier, 2011). The questionnaire consists of 24-items (e.g., When people cry in front of me I usually don't feel anything at all), and is subdivided into 6 subscales; kindness, indifference, common humanity, separation, mindfulness, and disengagement. Participants respond to items on a 5 -point Likert scale ( 1 almost never $\& 5$ almost always), with indif- 
ference, separation, and disengagement items reverse-scored. Cronbach alpha's for overall scale $\alpha=.90$, with kindness $\alpha=.77$, indifference $\alpha=.68$, common humanity $\alpha=.70$, separation $\alpha=.64$, mindfulness $\alpha=.67$, and disengagement $\alpha$ $=.57$.

Subjective Happiness Scale (SHS) (Lyubomirsky \& Lepper, 1999; Karakasidou, Pezirkianidis, Stalikas, \& Galanakis, 2016). The questionnaire was used in order to examine the subjectivity of persons' global happiness using four items rated on a 7-point Likert scale with higher scores reflecting greater happiness. Two items ask respondents to characterize themselves using both absolute ratings and ratings relative to peers, whereas the other two items offer brief descriptions of happy and unhappy individuals and ask respondents the extent to which each characterization describes them. Test-retest and self-peer correlations have suggested good to excellent reliability, and construct validation studies of convergent and discriminant validity have confirmed the use of this scale to measure the construct of subjective happiness (Lyubomirsky \& Lepper, 1999). Internal consistency reliability in the present study was $\alpha=.83$.

\subsection{Procedure}

The survey was online, as participants participated in the survey through an online platform created to collect data. At the beginning, an email was sent to each participant, which included an informative introductory note and an online link. The receivers of the e-mail after reading the introductory information note had the possibility through the e-mail link sent to them to be transferred to the online platform that included the information sheet and questionnaires. Through the information form, participants were informed about the purpose of the study and the context in which it is conducted. They were also informed about the anonymity and confidentiality of their data, the ability to withdraw from the process at any stage, and the fact that all information and data will be kept in privacy and will be used exclusively for research purposes. Additionally, the information sheet provided instructions for completing the questionnaires while finally, it is worth noting that the whole process followed ethical rules for conducting investigations.

At the completion of the questionnaires, the participants were asked to complete a demographic data package, the Compassion-for-others Scale (Pommier, 2011), the Professional Quality of Life Scale (Stamm, 2010), the Self-Compassion Scale (Neff, 2003a) as well as the Subjective Happiness Scale (Lyubomirsky \& Lepper, 1999). The process of completing the questionnaires lasted for about 15 20 minutes for each participant. The responses were automatically imported to the application database.

\section{Results}

For the purposes of this study, the results from raw data that were collected through the questionnaires were analyzed (see the attached SPSS file for raw da- 
ta). In particularly, the Means and Standard Deviations of self-compassion, subjective happiness, compassion for others and compassion fatigue were calculated for all participants. What is more, the correlations between them were analyzed in order to check for variables' inter-correlations indicating that correlations were generally within the small to medium range and they are presented in $\mathrm{Ta}$ ble 1 below.

For the first hypothesis of the study, in order to investigate the correlation between the levels of self-compassion and subjective happiness, a Pearson $(r)$ correlation analysis was applied to the data. A two-tailed Pearson's correlation revealed a strong, positive and significant correlation between self-compassion and subjective happiness $(r=.54, p<.001)$. More specifically, it was revealed a strong, positive and significant correlation between self-kindness and subjective happiness $(r=.53, p<.001)$, a medium, positive and significant correlation between common humanity and subjective happiness $(r=.40, p=.002)$ as well as a medium, negative and significant correlation between over-identified and subjective happiness $(r=-.49, p<.001)$, a medium, negative and significant correlation between self-judgment and subjective happiness $(r=-.40, p=.002)$ and a medium, negative and significant correlation between isolation and subjective happiness $(r=-.49, p<.001)$. No significant correlations were reported between mindfulness and subjective happiness $(r=.21, p=.106)$.

For the second hypothesis of the study, in order to investigate the correlation between the compassion fatigue and variables of self-compassion, compassion for others and subjective happiness of therapists a Pearson $(r)$ correlation analysis was applied to the data. A two-tailed Pearson's correlation revealed a low, negative and significant correlation between subjective happiness and compassion fatigue $(r=-.26, p=.05)$ as well as a medium, positive and significant correlation between compassion for others and compassion fatigue $(r=.32, p$ $=.013)$. No significant correlations were reported between self-compassion and compassion fatigue $(\mathrm{r}=-.16, p=.215)$.

For the third hypothesis of the study, hierarchical multiple regression analysis was used to investigate to what extent subjective happiness can be interpreted by

Table 1. Mean scores, standard deviations (SDs) and bivariate correlations between the variables $(N=58)$.

\begin{tabular}{ccccc}
\hline & $\begin{array}{c}\text { Subjective } \\
\text { happiness }\end{array}$ & Self-compassion & $\begin{array}{c}\text { Compassion } \\
\text { for others }\end{array}$ & $\begin{array}{c}\text { Compassion } \\
\text { fatigue }\end{array}$ \\
\hline Subjective happiness & - & $.54^{* *}$ & .02 & $-.26^{*}$ \\
Self-compassion & & - & $.43^{* *}$ & -.16 \\
Compassion for others & & & - & $.32^{\star}$ \\
Compassion fatigue & 19.86 & 89.93 & 104.55 & - \\
$M$ & 4.44 & 16.91 & 8.95 & 3.10 \\
\multicolumn{1}{c}{$S D$} & & & & \\
\hline
\end{tabular}

Note. ${ }^{*}<.05,{ }^{* *} p<.001$. 
the presence of the levels of self-compassion, compassion for others and compassion fatigue. For this purpose, the indicator $\mathrm{r}^{2}$ was used. Through the use of this indicator, we are informed about the common percentage of variance interpreted by the above variables. The results, as presented in Table 2 below, have shown a significant regression equation $(F(13,44)=6.15, p<.001)$, while the overall variance in subjective happiness interpreted by the predictive variables is $64 \%\left(R^{2}=.64\right)$. More specifically, the influence of self-kindness was found statistically significant, revealing the most powerful impact on subjective happiness $($ beta $=.72, t(44)=3.49, p<.001)$ with isolation $($ beta $=-.63, t(44)=-3.40, p$ $<.001)$, separation (beta $=-.42, t(44)=-3.28, p=.002)$, mindfulness compassion $($ beta $=.45, t(44)=2.00, p=.052)$ and disengagement $($ beta $=.39, t(44)=$ $2.64, p=.011)$ to follow.

For the forth hypothesis of the study, in order to investigate the correlation between the age, the years of professional experience, the average number of clients for all the therapists and study's variables, Pearson $(r)$ correlation analyses were applied to the data. Three two-tailed Pearson's correlations revealed a medium, negative and significant correlation between the age of the mental health professionals and compassion fatigue $(r=-.32, p=.015)$, no significant correlations $(p>.05)$ were revealed between the years of professional experience and variables of the study (subjective happiness, self-compassion, compassion for others and compassion fatigue) while a medium, positive and significant correlation between the average number of clients and compassion for others ( $r$ $=.41, p=.002)$ as well as compassion fatigue $(r=-.48, p<.001)$ was also calculated. Finally, an independent samples t-test showed that therapists who attend personal psychotherapy in the present reported higher levels of compassion for others $(M=107.28, S D=6.50)$ than the therapists who attended personal psychotherapy in the past $(\mathrm{M}=101.28, \mathrm{SD}=10.19), t(54)=2.63, p=.043$, at $a$ $=.05$. Additionally, it was also found that therapists who attend personal psychotherapy in the present reported higher levels of self-compassion ( $M=92.93$, $\mathrm{SD}=16.61)$ than the therapists who attended personal psychotherapy in the past $(\mathrm{M}=86.07, \mathrm{SD}=17.10)$, but however this difference shows that there is a tendency of change, as it was not statistically significant $(p>.05)$.

Table 2. The impact of self-kindness, isolation, separation, mindfulness compassion and disengagement on subjective happiness.

\begin{tabular}{cccccc}
\hline \multirow{2}{*}{ Criterions } & \multicolumn{5}{c}{ Subjective Happiness } \\
\cline { 2 - 6 } & $\mathrm{B}$ & SE & $\beta$ & $R^{2}$ & $F$ \\
\hline Self-Kindness & .85 & .24 & .72 & .64 & 6.15 \\
Isolation & -.76 & .22 & -.63 & .64 & 6.15 \\
Separation & -.78 & .24 & -.42 & .64 & 6.15 \\
Mind. Compass. & 1.05 & .53 & .45 & .64 & 6.15 \\
Disengagement & .80 & .30 & .39 & .64 & 6.15 \\
\hline
\end{tabular}




\section{Discussion}

The purpose of this research was to study the relationship between self-compassion, compassion for others, compassion fatigue, and subjective happiness for mental health specialists, who work as therapists. In particular, this study was examined the subjective happiness, a concept that refers to a set of positive characteristics that provide individuals with a positive adaptation in order to cope with demanding situations (Kumcagiz \& Gunduz, 2016). In other words, it was investigated the potential factors that can predict the high levels of subjective happiness to therapists as well as the potential protective role of subjective happiness in the negative effects of therapists' compassion fatigue. As predictors of the therapists' subjective happiness, were tested the self-compassion, which refers to a positive way of dealing with oneself in moments of pain or failure (Neff, 2003b) and also the compassion for others, which refers to "the emotion that arises when witnessing another's suffering and that subsequently motivates a desire to help" (Lopez, Sanderman, Ranchor, \& Schroevers, 2018). Moreover, subjective happiness and its relationship with compassion fatigue, which means the occupational burnout and secondary traumatic stress that therapists may experience (Figley, 2002; Stamm, 2010), was examined.

The results of the research confirmed the first hypothesis, according to which a positive correlation between self-compassion and subjective happiness of the mental health professionals was hypothesized. In particular, a positive correlation between self-compassion and subjective happiness was found, which means that the therapists who reported higher levels of self-compassion, reported higher levels of subjective happiness, a finding that is in line with the existing literature, linking self-compassion with positive mental health outcomes. More specifically, surveys that explored the concept of self-compassion in the general population showed that individuals with high levels of self-compassion react less strongly to negative events, make less negative thoughts and experience fewer negative feelings for themselves when they think of stressful or negative states (Leary et al., 2007; Neff, 2003a; Neff, Kirkpatrick, \& Rude, 2007). Alongside, the investigations that have studied the concept in a sample of therapists, showed that self-compassion is associated with higher levels of mental well-being (Patsiopoulos \& Buchanan, 2011). Generally, studies that examine self-compassion in both the general population and mental health experts argue that there are many positive results arising from the adoption of a self-compassionate attitude by suggesting that self-compassion can work protective in difficult, stressful and negative situations (Gilbert, 2009; Neff, Kirkpatrick, \& Rude, 2007; Patsiopoulos \& Buchanan, 2011; MacBeth \& Gumley, 2012).

These findings can be explained by the fact that individuals with self-compassion do not criticize themselves for failure, are more able to admit their mistakes, to modify non-productive behaviors, and to take on new challenges (Neff, 2009b). On the other hand, people who do not compassionate themselves when experiencing an unpleasant experience tend to criticize them, feel isolated from oth- 
ers, and absorb the negative experience and negative emotions, while at the same time, they choose strategies to manage negative events that are less effective than those used by people who compassionate themselves. Another hypothesis that can explain the positive relationship between self-compassion and subjective happiness is that therapists with higher levels of self-compassion are more likely to maintain an attitude that facilitates their involvement in self-care practices, as it has been shown that self-compassion facilitates the adoption of attitude that care and self-compassion is important (Neff, 2003b; Patsiopoulos \& Buchanan, 2011). Both the findings of this research and similar previous research findings highlight the importance of self-compassion and suggest its value in the developing aspects of positive psychology and professional wellbeing of therapists.

The results also verified the second hypothesis of the study, according to which a negative correlation between compassion fatigue and subjective happiness of mental health professionals was expected. Particularly, the therapists who reported higher levels of compassion fatigue reported lower levels of subjective happiness. This can be explained by the literature highlighting the findings revealing that the everyday stressors of the work's therapists can lead to significant harmful effects on their mental health and their ability to work effectively (Barnett, Baker, Elman, \& Schoener, 2007). In other words, due to the professionals' exposure to extremely stressful or traumatic events through their work may result to compassion fatigue, a decrease in the capacity or interest in the therapist's suffering the pain of others, and consequently to lower reported levels regarding their happiness (Adams, Boscarino, \& Figley, 2006; Bride \& Figley, 2007; Figley, 2002). So, the subjective happiness of the therapists seems to have an effect of reducing the fatigue of compassion they are experiencing and thus it constitutes a protective factor for therapists by mitigating the negative effects of compassion fatigue that they may experience, those of burnout and secondary traumatic stress.

Moreover, a positive correlation between compassion fatigue and compassion for others as well as no significant correlations were indicated between compassion fatigue and self-compassion. This finding can be explained, according to the theory, by the fact that the higher compassion for others a mental health professional may experience the more compassion fatigue levels he may presents depending on the exposure and stressful situation he is called to cope with (Beaumont, Durkin, Hollins Martin, \& Carson, 2016). The non significant correlations between compassion fatigue and self-compassion cannot abolish the overall theory for the positive and beneficial effects of the general concept of self-compassion. Nevertheless, this particular study is subject to restrictions and limitations which are going to, thoroughly, present below.

The results also confirmed the third hypothesis of the study, according to which higher levels of self-compassion and higher levels of compassion for others would predict higher levels of subjective happiness to therapists. The hierarchical multiple regression analysis that has been conducted showed that 
self-compassion and compassion for others experienced by therapists have an impact on the development of their subjective happiness. This finding highlights the importance of the concepts of self-compassion and compassion for others, as well as the role that they play in the happiness levels of the mental health professionals. An interpretation of these results is that self-compassion and compassion for others are core elements of the humanistic professions and therefore act protectively to the mental and occupational health of therapists, contributing positively to their subjective happiness.

The results of the forth hypothesis revealed significant information regarding the correlation between the age, the years of professional experience, the average number of clients for the therapists and the study's variables. It was found that compassion fatigue is negatively correlated with the professional's age, which means that as the age of therapist increases the compassion fatigue decreases. This finding can be interpreted by the fact that as the mental health professional grows up he manages to find the most appropriate and effective ways in order to cope better with his challenging and demanding profession. It was also revealed by the study that the years of professional experience do not have an effect on self-compassion, compassion for others, compassion fatigue and subjective happiness while on the other hand it was proved that the more clients a therapist attend the more compassion for others and compassion fatigue he experiences. The average number of clients seems to be a factor that should be taken into consideration by the therapists and may help them identify some of the causes that may have difficulty and hence to make a more immediate and effective solution. Finally, this particular hypothesis showed that the professionals who attend psychotherapy in the present report higher levels of compassion for others in comparison with those who attended psychotherapy in the past. This finding suggest that the mental health professional who continuously explores himself has a positive impact on his profession as he manages to be closer and more effective for his clients.

In conclusion, the findings of this research and the results of previous research can suggest that self-compassion has a protective function for the therapists by enhancing their subjective happiness, which in turn protects mental health and professional quality of life of therapists, as it plays an important role in reducing the negative effects of compassion fatigue.

\subsection{Implications}

The knowledge derived from this study could enrich the theory and practice around the supervision and personal therapy of professional counselors and therapists, through the recognition of the risks involved in their work, but also through the development of positive concepts that could protect them from these dangers. In addition, the findings from this research could be implicated in the development of new approaches to counseling and psychotherapy training programs. In particular, the results of this study showed that the therapists who had higher levels of self-compassion and compassion for others reported higher 
levels of subjective happiness and those with higher levels of subjective happiness reported lower levels of compassion fatigue, which means lower levels of burnout and secondary traumatic stress. The above knowledge can lead to the development of continuing educational programs aimed at enhancing the self-compassion of therapists and trainee therapists by informing them and training them in strategies for developing self-compassion.

Furthermore, the finding of this particular study that self-kindness and mindfulness compassion can predict subjective happiness of the mental health professionals and also the knowledge that has emerged about the importance of self-compassion to the therapists, helps to suggest ways in which the training program can be enriched to equip learners with tools and strategies to help them cope with the challenges of the profession more efficiently. The introduction of interventions aimed at promoting the self-compassion of trainees, through the development of individual resources and skills, is particularly important for the development of a new generation of professionals who can cope with an especially stressful job.

One way of enhancing a self-compassionate attitude of therapists and potential therapists is to enrich education programs with information and education in self-care practices that can help reduce the symptoms of compassion fatigue while at the same time boosting positive mental health factors wellness, such as self-compassion and subjective happiness but also the professional quality of life. The importance of self-care practices and their relationship to the above concepts has been supported in several studies (Neff, 2003b; Patsiopoulos \& Buchanan, 2011). Meditation is a practice of self-care and at the same time a strategy of developing compassion for oneself and others and has been proved to be helpful for professionals experiencing burnout, stress, anxiety, depression, low levels of job satisfaction and a sense of reduced professional self-efficacy (Shapiro, Austin, Bishop, \& Cordova, 2005) while meditation has also been associated with higher levels of self-compassion (Boellinghaus, Jones, \& Hutton, 2013; Neff, 2003b). Other strategies that have been found to contribute to self-compassion, or on some of its components, are the education in mindfulness based on stress reduction ("Mindfulness Based Stress Reduction training") (Shapiro et al., 2007) and compassionate education (“Compassionate Mind Training”) (Gilbert, 2009).

\subsection{Limitations and Future Research Directions}

Future research is useful to try to ensure a larger number of male participants, something that has not been done in this research. In addition, it would be especially useful to further explore specific practices and strategies that enhance self-compassion, compassion for others and subjective happiness, which will lead to the development of concrete interventions to strengthen them. It would be also important for future research not to be limited only to quantitative methodology but to carry out qualitative studies or proceed alternative multi-method ways to explore the above concepts, as the utilization of self-report measures come with both advantages and disadvantages. Self-report question- 
naires offer some insight to the inner experiences of individuals that could not be obtained by other measures (Paulhus \& Vazire, 2007). In addition, the variables of compassion and subjective happiness are personal emotional experiences that do not necessarily lead to observable reactions. Nonetheless, self-reports are subjected to bias (Paulhus \& Vazire, 2007).

One of the limitations of this research concerns the fact that sampling was not random but carried out with the opportunity method. A further limitation of the research is the small number of participants, as well as the much smaller number of male participants (6.9\%) compared to women (93.1\%). Also, the measurement of the research variables was done using self-report tools, which tends to increase the subjectivity of the measurements. Another important limitation of research concerns the probability of prejudice of the sample that has to do with the therapists who chose and those who did not choose to participate in the study. More specifically, therapists who eventually chose to participate in the research, by completing the questionnaires sent to them electronically, may have been interested in the subject studied. On the other hand, those therapists who may experience higher levels of stress, anxiety and burnout, may not have the interest to fill in the questionnaires or may have resistance to the research.

In addition, as the sample of the study consisted exclusively of mental health professionals living and working in Athens with $100 \%$ Greek ethnic composition, it leads to the fact that our results might not be generalizable to other groups and populations with quite different characteristics and thus the generalization concerns only the populations we have investigated. Finally, it may have been of great importance to examine in future studies the relationship of the variables of the present study to other variables such as the variety if the therapists psychotherapeutic approaches in order to investigate whether there would be any significant changes on the study's outcomes based on the different kind of approaches.

\subsection{Summary}

Therapists usually have the intense desire and goal to help other people cope with their difficulties. However, they are everyday faced with many challenges that can lead them to negative effects on their mental health and professional efficiency. The above research highlights the importance of positive factors of self-compassion, compassion for others and subjective happiness among therapists, as it has been found that the above variables are positively correlated and negatively with the compassion fatigue, which includes professional burnout and secondary traumatic stress. Their important role in mental health and professional quality of therapists is also evidenced by the fact that components of self-compassion and compassion for others are predictive factors for subjective happiness of the therapists while subjective happiness is a core factor for the low levels of compassion fatigue that professionals may experience. The present research can help to recognize the importance of developing self-care skills that will enhance the subjective happiness of the specialists, their self-compassionate 
attitude and the compassion for their clients. The development of these concepts may consist of a tool for counseling and psychotherapy professionals, providing them with the tools they need to cope with the demanding and stressful profession, to gain satisfaction and pleasure through it and consequently be as effective as possible with their clients.

\section{Conflicts of Interest}

The authors declare no conflicts of interest regarding the publication of this paper.

\section{References}

Adams, K. B., Matto, H. C., \& Harrington, D. (2001). The Traumatic Stress Institute Belief Scale as a Measure of Vicarious Trauma in a National Sample of Clinical Social Workers. Families in Society, 82, 363-371. https://doi.org/10.1606/1044-3894.178

Adams, R. E., Boscarino, J. A., \& Figley, C. R. (2006). Compassion Fatigue and Psychological Distress among social Workers: A Validation Study. American Journal of Orthopsychiatry, 76, 103-108.

Barnard, L. K., \& Curry, J. F. (2011). Self-Compassion: Conceptualizations, Correlates, \& Interventions. Review of General Psychology, 15, 289-303. https://doi.org/10.1037/a0025754

Barnett, J. E., Baker, E. K., Elman, N. S., \& Schoener, G. R. (2007). In Pursuit of Wellness: The Self-Care Imperative. Professional Psychology: Research and Practice, 38, 603-612. https://doi.org/10.1037/0735-7028.38.6.603

Beaumont, E., Durkin, M., Hollins Martin, C. J., \& Carson, J. (2016). Measuring Relationships between Self-Compassion, Compassion Fatigue, Burnout and Wellbeing in Trainee Counselors and Trainee Cognitive Behavioral Psychotherapists: A Quantitative Survey. Counseling and Psychotherapy Research, 16, 15-23.

https://doi.org/10.1002/capr.12054

Boellinghaus, I., Jones, F. W., \& Hutton, J. (2013). Cultivating Self-Care and Compassion in Psychological Therapists in Training: The Experience of Practicing Loving-Kindness Meditation. Training and Education in Professional Psychology, 7, 267-277. https://doi.org/10.1037/a0033092

Bride, B. E., \& Figley, C. R. (2007). The Fatigue of Compassionate Social Workers: An Introduction to the Special Issue on Compassion Fatigue. Clinical Social Work Journal, 35, 151-153. https://doi.org/10.1007/s10615-007-0093-5

Dunkley, J., \& Whelan, T. A. (2006). Vicarious Traumatization: Current Status and Future Directions. British Journal of Guidance \& Counseling, 34, 107-116. https://doi.org/10.1080/03069880500483166

Figley, C. R. (2002). Compassion Fatigue: Psychotherapists' Chronic Lack of Self Care. Journal of Clinical Psychology, 58, 1433-1441. https://doi.org/10.1002/jclp.10090

Germer, C. K., \& Neff, K. D. (2013). Self-Compassion in Clinical Practice. Journal of Clinical Psychology, 69, 856-867. https://doi.org/10.1002/jclp.22021

Gilbert, P. (2009). The Compassionate Mind: A New Approach to Life Challenges. London: Constable and Robinson Ltd.

Grant, L., \& Kinman, G. (2012). Enhancing Wellbeing in Social Work Students: Building Resilience in the Next Generation. Social Work Education, 31, 605-621.

https://doi.org/10.1080/02615479.2011.590931 
Heffernan, M., Quinn Griffin, M. T., McNulty, S. R., \& Fitzpatrick, J. J. (2010). Self-Compassion and Emotional Intelligence in Nurses. International Journal of Nursing Practice, 16, 366-373. https://doi.org/10.1111/j.1440-172X.2010.01853.x

Karakasidou, E., Pezirkianidis, C., Galanakis, M., \& Stalikas, A. (2017). Validity, Reliability and Factorial Structure of the Self Compassion Scale in the Greek Population. Journal of Psychology and Psychotherapy, 7, 313.

Karakasidou, E., Pezirkianidis, C., Stalikas, A., \& Galanakis, M. (2016). Standardization of the Subjective Happiness Scale (SHS) in a Greek Sample. Psychology, 7, 1753-1765. https://doi.org/10.4236/psych.2016.714164

Kraut, R. (1979). Two Conceptions of Happiness. Philosophical Review, 87, 167-196. https://doi.org/10.2307/2184505

Krieger, T., Hermann, H., \& Zimmermann, J., \& Holtforth, M. G. (2015). Associations of Self-Compassion and Global Self-Esteem with Positive and Negative Affect and Stress Reactivity in Daily Life : Findings from a Smart Phone Study. Personality and Individual Differences, 87, 288-292. https://doi.org/10.1016/j.paid.2015.08.009

Kumcagiz, H., \& Gunduz, Y. (2016). Relationship between Psychological Well-Being and Smartphone Addiction of University Students. International Journal of Higher Education, 5, 144-156. https://doi.org/10.5430/ijhe.v5n4p144

Lawson, G., \& Myers, J. E. (2011). Wellness, Professional Quality of Life, and Career Sustaining Behaviors: What Keeps Us Well? Journal of Counseling \& Development, 89, 163-171. https://doi.org/10.1002/j.1556-6678.2011.tb00074.x

Leary, M. R., Tate, E. B., Adams, C. E., Allen, A. B., \& Hancock, J. (2007). Self-Compassion and Reactions to Unpleasant Self-Relevant Events: The Implications of Treating Oneself Kindly. Journal of Personality and Social Psychology, 92, 887-904. https://doi.org/10.1037/0022-3514.92.5.887

Lopez, A., Sanderman, R., Ranchor, A. V., \& Schroevers, M. J. (2018). Compassion for Others and Self-Compassion: Levels, Correlates, and Relationship with Psychological Well-Being. Mindfulness, 9, 325-331. https://doi.org/10.1007/s12671-017-0777-z

Lyubomirsky, S. (2001). Why Are Some People Happier than Others? The Role of Cognitive and Motivational Processes in Wellbeing. American Psychologist, 56, 239-249. https://doi.org/10.1037/0003-066X.56.3.239

Lyubomirsky, S., \& Lepper, H. S. (1999). A Measure of Subjective Happiness: Preliminary Reliability and Construct Validation. Social Indicators Research, 46, 137-155. https://doi.org/10.1023/A:1006824100041

MacBeth, A., \& Gumley, A. (2012). Exploring Compassion: A Meta-Analysis of the Association between Self-Compassion and Psychopathology. Clinical Psychological Review, 32, 545-552. https://doi.org/10.1016/j.cpr.2012.06.003

Myers, D. G., \& Diener, E. (1995). Who Is Happy? Psychological Science, 6, 10-19. https://doi.org/10.1111/j.1467-9280.1995.tb00298.x

Neely, M. E., Schallert, D. L., Mohammed, S. S., Roberts, R. M., \& Chen, Y. (2009). Self-Kindness When Facing Stress: The Role of Self-Compassion, Goal Regulation, and Support in College Students' Well-Being. Motivation Emotion, 33, 88-97. https://doi.org/10.1007/s11031-008-9119-8

Neff, K. D. (2003a). Self-Compassion: An Alternative Conceptualization of a Healthy Attitude toward Oneself. Self and Identity, 2, 85-101. https://doi.org/10.1080/15298860309032

Neff, K. D. (2003b). The Development and Validation of a Scale to Measure Self-Compassion. Self and Identity, 2, 223-250. 
https://doi.org/10.1080/15298860309027

Neff, K. D. (2009a). Self-Compassion. In M. R. Leary, \& R. H. Hoyle (Eds.), Handbook of Individual Differences in Social Behavior (pp. 561-573). New York: Guilford Press.

Neff, K. D. (2009b). The Role of Self-Compassion in Development: A Healthier Way to Relate to Oneself. Human Development, 52, 211-214. https://doi.org/10.1159/000215071

Neff, K. D., \& Vonk, R. (2009). Self-Compassion versus Global Self-Esteem: Two Different Ways of Relating to Oneself. Journal of Personality, 77, 23-50. https://doi.org/10.1111/j.1467-6494.2008.00537.x

Neff, K. D., Kirkpatrick, K., \& Rude, S. S. (2007). Self-Compassion and Its Link to Adaptive Psychological Functioning. Journal of Research in Personality, 41, 139-154. https://doi.org/10.1016/j.jrp.2006.03.004

Patsiopoulos, A. T., \& Buchanan, M. J. (2011). The Practice of Self-Compassion in Counseling: A Narrative Inquiry. Professional Psychology: Research and Practice, 42, 301-307. https://doi.org/10.1037/a0024482

Paulhus, D. L., \& Vazire, S. (2007). The Self-Report Method. In R. W. Robins, R. C. Fraley, \& R. F. Krueger (Eds.), Handbook of Research Methods in Personality Psychology (pp. 224-239). London: The Guilford Press.

Pommier, E. A. (2011). The Compassion Scale. Dissertation Abstracts International Section A: Humanities and Social Sciences, 72, 1174.

Ringenbach, R. (2009). A Comparison between Counselors Who Practice Meditation and Those Who Do Not on Compassion Fatigue, Compassion Satisfaction, Burnout and Self-Compassion. Akron, OH: Doctoral Dissertation, University of Akron.

Rosenberg, T., \& Pace, M. (2006). Burnout among Mental Health Professionals: Special Considerations for the Marriage and Family Therapist. Journal of Marital and Family Therapy, 32, 87-99. https://doi.org/10.1111/j.1752-0606.2006.tb01590.x

Rupert, P. A., \& Morgan, D. J. (2005). Work Setting and Burnout among Professional Psychologists. Professional Psychology: Research and Practice, 36, 544-550. https://doi.org/10.1037/0735-7028.36.5.544

Sbarra, D. A., Smith, H. L., \& Mehl, M. R. (2012). When Leaving Your Ex, Love Yourself: Observational Ratings of Self-Compassion Predict the Course of Emotional Recovery Following Marital Separation. Psychological Science, 23, 261-269. https://doi.org/10.1177/0956797611429466

Seligowski, A. V, Miron, L. R., \& Orcutt, H. K. (2014). Relations among Self-Compassion, PTSD Symptoms, and Psychological Health in a Trauma-Exposed Sample. Mindfulness, 6, 1033-1041.

Shapiro, S. L., Astin, J. A., Bishop, S. R., \& Cordova, M. (2005). Mindfulness-Based Stress Reduction for Health Care Professionals: Results from a Randomized Trial. International Journal of Stress Management, 12, 164-176. https://doi.org/10.1037/1072-5245.12.2.164

Shapiro, S. L., Brown, K. W., \& Biegel, G. M. (2007). Teaching Self-Care to Caregivers: Effects of Mindfulness-Based Stress Reduction on the Mental Health of Therapists in Training. Training and Education in Professional Psychology, 1, 105-115. https://doi.org/10.1037/1931-3918.1.2.105

Skovholt, T. M., \& Ronnestad, M. H. (2003). Struggles of the Novice Counselor and Therapist. Journal of Career Development, 30, 45-58. https://doi.org/10.1177/089484530303000103

Skovholt, T. M., Grier, T. L., \& Hanson, M. R. (2001). Career Counseling for Longevity: 
Self-Care and Burnout Prevention Strategies for Counselor Resilience. Journal of Career Development, 27, 167-176. https://doi.org/10.1177/089484530102700303

Smith, P. L., \& Moss, S. B. (2009). Psychologist Impairment: What Is It, How Can It Be Prevented, and What Can Be Done to Address It? Clinical Psychology: Science and Practice, 16, 1-15.

Stamm, B. H. (2005). The Pro-QOL Manual: The Professional Quality of Life Scale: Compassion Satisfaction, Burnout \& Compassion Fatigue/Secondary Trauma Scales. Baltimore, MD: Sidran Press.

Stamm, B. H. (2010). The Concise ProQOL Manual(2nd ed.). Pocatello, ID: ProQOL.org.

Van Dam, N. T., Sheppard, S. C., Forsyth, J. P., \& Earleywine, M. (2011). Self-Compassion Is a Better Predictor than Mindfulness of Symptom Severity and Quality of Life in Mixed Anxiety and Depression. Journal of Anxiety Disorders, 25, 123-130. https://doi.org/10.1016/j.janxdis.2010.08.011

Wei, M., Liao, K. Y., Ku, T., \& Shaffer, P. A. (2011). Attachment, Self-Compassion, Empathy, and Subjective Well-Being among College Students and Community Adults. Journal of Personality, 79, 191-221. https://doi.org/10.1111/j.1467-6494.2010.00677.x

Wren, A. A., Somers, T. J., Wright, M. A., Goetz, M. C., Leary, M. R., Fras, A. M., Huh, B. K., Rogers, L. L., \& Keefe, F. J. (2012). Self-Compassion in Patients with Persistent Musculoskeletal Pain: Relationship of Self-Compassion to Adjustment to Persistent Pain. Journal of Pain and Symptom Management, 43, 759-770.

https://doi.org/10.1016/j.jpainsymman.2011.04.014

Yang, X. (2016). Self-Compassion, Relationship Harmony, versus Self-Enhancement: Different Ways of Relating to Well-Being in Hong Kong Chinese. Personality and Individual Differences, 89, 24-27. https://doi.org/10.1016/j.paid.2015.09.006 